Voix et Images

voixetimages

\title{
L'auteur implicite dans Trente arpents : modes de présence et signification narrative
}

\section{Agnès Whitfield}

Volume 8, numéro 3, printemps 1983

Jacques Ferron

URI : https://id.erudit.org/iderudit/200404ar

DOI : https://doi.org/10.7202/200404ar

Aller au sommaire du numéro

Éditeur(s)

Université du Québec à Montréal

ISSN

0318-9201 (imprimé)

1705-933X (numérique)

Découvrir la revue

\section{Citer cet article}

Whitfield, A. (1983). L'auteur implicite dans Trente arpents : modes de présence et signification narrative. Voix et Images, 8(3), 485-494.

https://doi.org/10.7202/200404ar d'utilisation que vous pouvez consulter en ligne.

https://apropos.erudit.org/fr/usagers/politique-dutilisation/ 


\title{
L'auteur implicite dans Trente arperits: modes de présence et signification narrative
}

\author{
par Agnès Whitfield, Université Queen's
}

La présence de l'auteur dans Trente arpents a fait l'objet de nombreuses études fort divergentes. Tantôt on souligne le réalisme, l'objectivité de Ringuet, en attribuant une valeur proprement documentaire à son oeuvre.' Tantót on déclare qu'il verse plutôt dans la caricature, voire qu'il "salit honteusement le chaste paysan du Québec". ${ }^{2}$ Toutefois, aussi contradictoires qu'ils soient, ces jugements se fondent tous sur une appréciation essentiellement mimétique de l'optique narrative choisie par Ringuet. Si le rapport perçu par les critiques, entre le portrait du terroir dressé par Ringuet et la réalité du paysan québécois, varie de la correspondance la plus étroite à la totale dissemblance, le critère d'évaluation n'en demeure pas moins, pour tous, celui de la mimésis.

Le plus souvent, il s'agit là d’une démarche critique explicite. Aussi fait-on remarquer que Trente Arpents se distingue nettement des romans antérieurs de la terre, au Québec, parce que, par opposition à l'idéalisme d'un Patrice Lacombe ou d'un FélixAntoine Savard, Ringuet "occupe le poste d'observateur perspicace et du narrateur anonyme, notant les choses telles qu'il les voit, dépeignant ses personnages tels qu'ils sont". " A M. Servais-Maquoi de renchérir: "Ringuet ne cherche ni à embeilir, ni à assombrir le monde paysan qu'il regarde évoluer: dans Trente Arpents, la fiction est fort près de s'identifier à la réalité décrite". "Cette même approche critique domine également, mais d'une manière plus subtile, les études récentes, moins normatives, qui tentent de cerner la vision du monde de Ringuet à travers une analyse de ses commentaires sur le mode de vie de ses personnages. ${ }^{5}$

Or, sans mettre en doute l'intérêt du contenu mimétique de la présence textuelle de l'auteur, que nous appellerons plutôt l'auteur implicite pour distinguer une présence uniquement textuelle de l'auteur réel, ${ }^{6}$ il nous semble que la forme de cette présence mérite tout aussi bien d'être analysée. Comme le souligne W. Booth dans son livre sur la Rhetorique de la fiction, la présence de l'auteur implicite peut prendre maintes formes différentes. Sans tenir compte des caractéristiques qu'il révèle de luimême par le genre d'anecdotes et de personnages qu'il décrit, l'auteur implicite se manifeste, en tant qu'instance narrative, par les adresses directes au lecteur, les commentaires et jugements sur les personnages, les changements de point de vue à l'intérieur de l'oeuvre, et les raccourcis temporels ou plus généralement tous les artifices 
organisateurs du récit. ${ }^{7}$ Dans cette multiplicité de possibles, les formes de présence réalisées dans un texte donné ne peuvent résulter que de choix et elles contribuent ainsi à l'articulation de la signification dans le texte. Plus précisément, les différentes manifestations de l'auteur implicite constituent autant d'appels à un lecteur virtuel ou narrataire et informent en ce sens la structure narrative du roman. Cela est particulièrement vrai dans un roman comme Trente arpents où, en l'absence d'un narrateurpersonnage, c'est à l'auteur implicite en tant que narrateur anonyme, ${ }^{8}$ qu'incombe seul la responsabilité narrative du texte.

Pour les théoriciens traditionnels du point de vue, il s'agit là surtout d'une question de fins et de moyens, l'auteur optant pour telle forme de présence en fonction de tel effet recherché. ${ }^{9}$ Cependant, pour respecter l'autonomie et le caractère dynamique du texte, nous préférons dire que les formes de présence de l'auteur implicite non seulement nous renseignent, par leurs effets, sur son intention auprès du lecteur, mais elles la concrétisent, la manifestent. Autrement dit, au lieu de précéder, consciente d'elle-même, la construction du texte, l'intention phatique-de l'auteur implicite se réalise mème au fur et à mesure que, par ses choix, il établit des rapports particuliers avec son narrataire. D'où la nécessité, pour suivre cette intention dynamique, de repérer à la fois les formes de présence choisies par l'auteur implicite et l'utilisation qu'il en fait au cours du texte. Voilà donc la double démarche que nous allons suivre maintenant, délaissant ainsi les interprétations mimétiques de Trente arpents pour nous mettre dans la perspective de son fonctionnement en tant que discours narratif.

Des multiples modes possibles de présence de l'auteur implicite, nous ne retiendrons aux fins de notre étude que ceux qui, par leur fréquence ou leur importance, se montrent les plus significatives pour la structure narrative de Trente arpents. Pour faciliter l'analyse, nous les grouperons sous deux rubriques, suivant qu'elles sont d'ordre explicite, c'est-z-dire qu'elles constituent un appel direct au narrataire, ou, plutőt, de genre implicite, reposant sur des choix structuraux. Pour ce qui est des manifestations directes de l'auteur implicite, nous tiendrons donc compte des jugements sur les personnages, des descriptions visant le narrataire, des comparaisons, des négations, des questions rhétoriques et des allusions directes au narrataire au moyen de pronoms personnels (nous, vous, on) et d'adverbes (certes). Parmi les manifestations implicites de l'auteur, nous jugerons pertinents la durée narrative et les rapports d'ordre, au sens où $\mathrm{G}$. Genette emploie ces termes, ${ }^{10}$ ainsi que l'utilisation du dialogue rapporté.

Exception faite des dialogues rapportés, les manifestations implicites de l'auteur se distinguent des traces explicites par leur caractère obligatoire. Tout récit narratif reposant par definition sur une double temporalité, le narrateur doit établir une durée narrative ainsi que certains rapports d'odre entre la temporalité chronologique de l'aventure et la successivité paginale du texte. Par contre, il est libre non seulement de donner un contenu particulier aux manifestions explicites de sa présence, mais aussi de les supprimer complètement.

Cette observation, d'ordre quelque peu elémentaire, nous amène à une première constatation sur la structure narrative de Trente arpents, à savoir la très haute fréquence des manifestations explicites de l'auteur. Conséquence peut-être tout simplement de l'influence des modèles littéraires de l'époque, ce phénomène témoigne 
néanmoins de l'importance textuelle des rapports entre l'auteur implicite et son narra taire. Cependant, ce qui parait moins susceptible d'une explication conventionnelle, ce sont les variations frappantes dans la fréquence des manifestations ou marqueurs particuliers de ces rapports. Pour nous en tenir à des comptabilisations approximatives, nous relevons une baisse sensible du nombre de comparaisons, de jugements lou descriptionsl et de négations du début à la fin du texte. Par contre, les questions rhétoriques et les allusions directes au lecteur au moyen de pronoms et d'adverbes se multiplient. En général, ces différences de fréquence témoignent d'un abandon, par l'auteur implicite, de ses visées documentaires initiales en faveur de buts d'ordre plutót psychologique et elles se doublent d'un rapprochement de l'auteur implicite avec son personnage principal, Euchariste Moisan. Entrons maintenant dans le détail.

Comme on le sait, le roman se divise en quatre sections qui portent les noms des saisons et qui sont composées du même nombre de pages environ, à l'exception de la dernière section, plus courte, de l'hiver. Mais pour les 34 passages où l'auteur implicite intervient dans la section intitulee "Printemps", pour juger ses personnages ou décrire la vie paysanne, nous ne trouvons que 26 interventions équivalentes dans "I'Étë", 21 dans "I'Automne" et 13-dans "I'Hiver". Cette réduction du nombre d'intervention's s'accompagne d'un changement de valeur et de portée. Non seulement les interventions de l'auteur implicite tendent-elles à se raccourcir, de paragraphes entiers dans la première section du livre aux simples phrases dans la dernière, mais leur objèt aussi se modifie.

Dans la section "Printemps", l'auteur implicite intervient surtout pour peindre le milieu paysan. Nous y trouvons de nombreux passages comme celui-ci où le rôle qu'il cherche à jouer auprès du narrataire est surtout celui du documentaliste, voire du sociologue:

La population des paroisses suit une constante assez marquée dans le Québec: le nombre de familles terriennes varie peu, car, la division des terres répugne au paysan. Le père préfère en général voir ses fils puînés partir pour les terres neuves, laissant à l'aîné la possession indivise du bien familial, plutôt que le déchirer entre ses enfants."

Il en va de mème dans les passages, moins fréquents, où il décrit ses personna ges. La description sert alors davantage à mettre en relief les caractéristiques du type qu'à souligner des traits individuels:

lis parlaient lentement et peu, à leur accoutumée, étant paysans, donc chiches de parolẹs. Mais ils y mettaient aujourd'hui les hésitations, les tâtonnements qui conviennent lorsqu'on parle de choses de conséquence (p. 9).12

Mais dans la dernière section du livre, les jugements ou descriptions de l'auteur implicite ont presque toujours une portée psychologique. Plus précisément, ils servent à mettre en relief la personnalité d'Euchariste Moisan: "Ce qui le terrifiait, au début, c'était la crainte de s'endormir, de manquer un instant à son devoir de surveillance" (p. 282): "Mais Euchariste ne voyait rien. II n'avait rien répondu non plus, mais serrait les lèves. A tout prix il voulait endiguer le récit de ses malheurs, de sa déchéance" (p. 274). Si quelques interventions de l'auteur implicite gardent encore un élément universalısant, celui-ci porte moins sur le milieu paysan que sur la nature humaine: " $\mathrm{Ce}$ 
surtout qu'il ne pouvait supporter était la rencontre des voisins (...) Phydime, particulièrement, avec qui il se trouvait constamment nez à nez, qu'il voyait partout, comme: toujours les gens que l'on veut éviter" (p.229).

Si l'on se rappelle que la déchéance de Moisan et son exil malheureux aux ÉtatsUnis correspondent à un phénomène sociologique réel du milieu paysan décrit dans Trente arpents, il est significatif que l'auteur implicite choisit de mettre surtout en relief, par ses jugements, la stratégie personnelle de Moisan. Certes, le lecteur est libre d'accorder une valeur symbolique à celle-ci. II n'empêche que la transformation de la forme et de la portée des interventions de l'auteur implicite trahit une modification de l'intention de ce dernier auprès du lecteur. Au désir d'informer son narrataire, d'une manière directe et intellectuelle, succède l'intention de toucher autrui tout en l'informant. La communication immédiate donne lieu à un échange médiatisé où l'importance des personnages devient primordiale.

Bien qu'elle soit numériquement moins sensible, la réduction dans les néga tions et les comparaisons a le même effet. Dan's la première section du livre, nous comptons au moins $\mathbf{8}$ exemples de formulations négatives qui rappellent les jugements initiaux de l'auteur implicite par leur teneur généralisante. Aussi, à propos des conversations entre Euchariste Moisan et sa femme, nous lisons qu'ils " $n$ 'échangeaient pas des idées qui sont le papier-monnaie de l'esprit, bon pour les gens des villes, mais bien des faits qui sont les pièces de métal, les bonnes pièces d'or" (p. 12). L'auteur implicite s'interpose ainsi entre ses-personnages et son narrataire, pour souligner à celui-ci les éléments typiques du comportement de ceux-là.

Mais dans les parties du livre intitulées "Eté" et "Automne", la quantité de phra ses négatives tombe respectivement à 5 et a 3 , pour ne se chiffrer qu'à 2 dans la dernière section sur l'hiver. Elles perdent en même temps leur valeur didactique ou documentaire au profit d'un plus grand effet émotif (Euchariste "ne réfléchit pas que Raymond, de sa fenêtre, avait pu voir tout en s'habillant à loisir. La catastrophe qui jusquelà l'écrasait servit de tremplin à sa haine. Phydime était bien capable par jalousie d'avoir mis le feu à sa grange") (p. 200) ou dramatique ("Ce qu'Alphonsine ne dit pas, c'est qu'elle a vu l'oiseau de malheur frôler la tête de son homme") (p. 112).

N'allons pas croire qu'en abandonnant la négation, l'auteur implicite cesse de commenter les lapsus des personnages ou les virtualités non réalisées. Parallèlement au déclin de la négation, nous constatons une remontée du conditionnel antérieur qui permet ce genre de commentaire de la part de l'auteur implicite sans toutefois l'obliger, comme la négation, à sortir carrément de l'optique des personnages: "ll eût voulu lui parler cormme autrefois, de père en fils" (p. 124).

L'analyse des comparaisons est plus complexe. Sans parler d'une extension méta phorique, le terme peut englober plusieurs phénomènes différents qui, par leur intégration aux reseaux anaphoriques, indiciels, thématiques ou symboliques, relévent davantage de l'analyse du roman en tant qu'histoire ou anecdote que du niveau discursif du texte. Pour notre propos, cependant, la signification de la comparaison réside dans le fait qu'en dehors des passages où les personnages se parlent entre eux, elle évoque le dialogue entre l'auteur implicite et le lecteur virtuel. Cette évocation, implicite dans le cas des métaphores, est explicitée dans les comparaisons, par l'emploi 
de la conjonction "comme". Dans Trente arpents, l'incidence de ce mot baisse sensiblement au cours du texte, de 10 mentions dans la section initiale, a 5 dans "Eté", avant de se stabiliser à 3 dans "Automne" et "Hiver". En même temps, l'objet des comparaisons change. Si dans "Printemps", celles-ci portent surtout sur la terre ou des phénomènes météorologiques, dans les sections subséquentes, elles mettent plutôt en relief les traits de caractère des personnages ou leurs émotions: "Il lui venait (à Euchariste) surtout par moments une intolérable sensation d'éternité comme au voya geur perdu dans la forêt"' (p. 266).

Tout comme la réduction dans le nombre de jugements et de descriptions, la suppression progressive des comparaisons et des négations a pour effet d'accentuer le rôle médiateur des personnages, aux dépens du dialogue direct entre l'auteur implicite et son narrataire. Or, dans un tel contexte, l'importance croissante des questions rhétoriques et des allusions au narrataire, au moyen de pronoms personnels et d'adverbes, semble quelque peu paradoxale, d'autant plus que ces modes de présence sont de loin plus directes que les manifestations, somme toute assez subtiles, de l'auteur implicite que nous venons d'étudier. Seulement, pqur reprendre une distinction du formaliste russe. Tynianov, il ne faut pas confondre un élément formée d'une oeuvre littéraire, entendue comme système, avec sa "fonction constructive (...) sa possibjilité d'entrer en corrélation avec les autres éléments du même système". ${ }^{13}$ C'est-à-ditre que la fréquence croissante de certains marqueurs formels de la présence de l'auteur implicite ne vient pas forcément confirmer leur fonction phatique usuelle.

En effet, dans Trente arpents, la fonction des questions rhétoriques ne consiste que rarement à évoquer le dialogue entre l'auteur implicite et son narrataire. Bien plus souvent, et ce, dès le déburt du roman, elles associent le lecteur virtuel aux réflexions des personnages: "Les seuls souvenirs nets qu'il en avait gardés étaient d'une monta gne (...) Quoi encore? Ah! oui: le ruisseau et la pêche" (p. 9). La fréquence croissante des questions rhétoriques, qui passent de 15, dans les deux premières sections du roman, à 30 dans "Automne" et "Hivẹe", met donc surtout en valeur la communication entre personnages et narrataire. Cest encore le rôle médiateur des personnages qui se trouve renforcé. Mais, élément ṇquveau, l'ambiguitté de la prise en charge discursive de ces questions (on dirait que l'auteur se fond aux personnages pour les poserl, porte à croire que la fonction médiatrice des personnages repose essentiellement sur une fonte progressive de la perspective de l'auteur implicite et de celle des personnages.

Ce lien se précise lorsquẹ nous examinons la façon dont l'auteur implicite interpelle le lecteur virtuel. Nous savons que les pronoms personnels "on", "tu" et "vous" marquent souvent une allusion directe ("tu" ou "vous") ou indirecte ("on", "nous") de la part de l'auteur implicite à son narrataire. Dans Trente arpents, les marqueurs les plus fréquents sont le "vous" et le "on". Tandis que l'emploi du "vous" pour désigner le lecteur demeure relativement stable 12 mentions dans "Printemps" et 1 mention dans les trois sections subséquentes), l'utilisation du "on" s'accroit beaucoup (de 23 mentions dans les deux sections initiales a 34 mentions dans "Automne" et "Hiver"). A cette fréquence croissante correspond, toutefois, un changement important de fonction. 
de la conjonction "comme". Dans Trente arpents, l'incidence de ce mot baisse sensiblement au cours du texte, de 10 mentions dans la section initiale, a 5 dans "Eté", avant de se stabiliser à 3 dans "Automne" et "Hiver". En même temps, l'objet des comparaisons change. Si dans "Printemps", celles-ci portent surtout sur la terre ou des phẹnomènes météorologiques, dans les sections subséquentes, elles mettent plutôt en. relief les traits de caractère des personnages ou leurs émotions: "Il lui venait (à Euchariste) surtout par moments une intolérable sensation d'éternité comme au voya geur perdu dans la forêt" (p. 266).

Tout comme la réduction dans le nombre de jugements et de descriptions, la suppression progressive des comparaisons et des négations a pour effet d'accentuer le rôle médiateur des personnages, aux dépens du dialogue direct entre l'auteur impli; cite et son narrataire. Or, dans un tel contexte, l'importance croissante des questions rhétoriques et des allusions au narrataire, au moyen de pronoms personnels et d'adverbes, semble quelque peu paradoxale, d'autant plus que ces modes de présence sont de loin plus directes que les manifestations, somme toute assez subtiles, de l'auteur implicite que nous venons d'étudier. Seulement, pour reprendre une distinction du formaliste russe. Tynianov, il ne faut pas confondre un élément formée d'une oeuvre littéraire, entendue comme système, avec sa "fonction constructive (...) sa possibilité d'entrer en corrélation avec les autres éléments du mème système". ${ }^{13} \mathrm{C}^{\prime}$ est-àdire que la fréquence croissante de certains marqueurs formels de la présence de l'auteur implicite ne vient pas forcément confirmer leur fonction phatique usuelle.

En effet, dans Trente arpents, la fonction des questions rhétoriques ne consiste que rarement à évoquer le dialogue entre l'auteur implicite et son narrataire: Bien plus souvent, et ce, dès le début du roman, elles associent le lecteur virtuel aux réflexions des personnages: "Les seuls souvenirs nets qu'il en avait gardés étaient d'une montagne (...) Quoi encore? Ah! oui: le ruisseau et la pêche" (p. 9). La fréquence croissante des questions rhétoriques, qui passent de 15, dans les deux premières sections du roman, a 30 dans "Automne" et "Hiver", met donc surtout en valeur la communication entre personnages et narrataire. C'est encore le róle médiateur des personnages qui se trouve renforcé. Mais, élément nouveau, l'ambiguité de la prise en charge discursive de ces questions lon dirait que l'auteur se fond aux personnages pour les poser), porte à croire que la fonction médiatrice des personnages repose essentiellement sur une fonte progressive de la perspective de l'auteur implicite et de celle des personnages.

Ce lien se précise lorsque nous examinons la façon dont l'auteur implicite interpelle le lecteur virtuel. Nous savons que les pronoms personnels "on", "tu" et "vous" marquent souvent une allusion directe ("tu" ou "vous") ou indirecte ("on", "nous") de la part de l'auteur implicite à son narrataire. Dans Trente arpents, les marqueurs les plus fréquents sont le "vous" et le "on". Tandis que l"emploi dụ "vous" pour désigner le lecteur demeure relativement stable $(2$ mentions dans "Printemps" et 1 mention dans les trois sections subséquentes), l'utilisation du "on" s'accroît beaucoup (de 23 mentions dans les deux sections initiales a 34 mentions dans "Automne" et "Hiver"). A cette fréquence croissante correspond, toutefois, un changement important de fonction. 
Certes, il s'en fallait qu'Euchariste fût un jaboteur; mais il avait sur les lèvres un récit de la vie courante làbas, qui n'attendait qu'une question d'Ephrem pour sortir au grand jour de'la sympathie (p. 249 ).

Comme pour les questions rhétoriques, c'est le statut discursif ambigu de "certes" quil le relie tant au personnage principal (Euchariste Moisanl qu'à l'auteur implicite Que ce rápprochement se réalise toujours lorsqu'il est question d'un départ, d'un đésaccord ne semble guère dû à une simple coîncidence: Ón dirait plutốt que la fusion des perspectives de l'auteur implicite et de son héros repose sur une problématique commune, celle de la rupture. Les modes implicites de présence de l'auteur c'est-àdire ses choix structuraux en ce qui concerne la durée narrative, les rapports d'ordre, le codage temporel et le recours au dialogue rapporté, permettent-ils de vérifier cette hypothèse?

Les variations dans la durée narrative et le recours au dialogue rapporté confirment de façon générale l'importance croissante du rôle médiateur des personnages. Le pourcentage approximatif du texte consacré aux dialogues rapportés passe de $20 \%$ dans la section initiale à $27 \%$ dans "Hiver". En même temps, conformément à l'évolution des comparaisons et descriptions, l'objet de ces dialogues devient de plus ẹ plus psychologique aux dépens des discussions politiques, fort nombreuses dans les deux premières sections du roman. Quant à la durée narrative, que Genette définiț comme "le rapport entre une durée, celle de l'histoire, mesurée en secondes, minutes, heures, jours, mois et années et une longueur: celle du texte, mesurée en lignes et en pages" ${ }^{\prime 14}$ elle connait un ralentissement progressif tout à fait conforme à la mise en relief de la tragédie personnelle de Moisan. Ainsi, si dans "Été". I'auteur implicite résume sommairement vingt-deux ans environ de la vie de Moisan, ce chiffre tombe à seize ans dans "Automne" et, réduction radicale, à deux ans dans "Hiver". ${ }^{15}$ Parallèlement, là concordance entre les saisons de l'histoire et les titres des sections du roman s'ams' liore. C'est l'hiver qui domine la section initiale du livre, malgré son titre "Printemps", tandis que dans "Eté", sont racontés des événements tant de l'été (mort d'Alphonsine) que de l'hiver. Par contre, la section "Automne" concerne surtout l'époque des moissons, et "Hiver", le premier hiver d'Euchariste aux Ėtats-Unis. Tout comme l'évolution de ses modes de présence explicites, les choix structuraux de l'auteur implicite relatifs aux dialogues rapportés et à la durée narrative témoignent ainsi, sans aucun doute, de son rapprochement affectif avec Euchariste Moisan.

II ne reste maintenant qu'à confirmer les termes de ce rapprochement. Or, à cet égard, les variations dans le codage temporel et les rapports d'ordre choisis par l'auteur implicite sont particulièrement révélatrices. Dans l'ensemble, Trente arpents est un récit au passé, l'auteur implicite adoptant ce que Genette appelle la narration ultérieure. ${ }^{16}$ Le roman comporte pourtant quelques passages où, exceptionnellement, le récit est contemporain du temps de l'aventure sans toutefois que l'on puisse assimiler l'emploi du présent au présent historique. ${ }^{17}$ || s'agit plutôt d'un véritable changement dans la perspective temporelle de l'auteur implicite.

Dans la section initiale du livre, ce changement s'explique par ce que $\mathrm{H}$. Weinrich appelle le passage du monde narratif au monde commenté. ${ }^{18} L^{\prime}$ auteur implicite emploie le présent pour commenter, à l'intention de son lecteur, une certaine réalité 
sociologique l'La population des paroisses suit une constante assez marquée dans le Québec: le nombre de familles terriennes varie peu... (p. 51 )/ ou familiale ('Les épaules de Mélie sursautent et sa figure soudain relevée fige ses rides dans la lumière. (...) Pour Ephrem, c'est sa tante, quoiqu'il n'y ait entre eux qu'un bien lointain cousinage"' (p. 15-16). Le commentaire sociologique rejoint sans doute les objectifs documentaires initiaux de l'auteur implicite. Cependant, la description, faite au présent et non pas, comme c'est d'habitude le cas, à l'imparfait, du milieu familial, incomplet, d'Euchariste Moisan semble présager la problématique de la rupture qui relie plus tard l'auteur implicite et son protagóniste.

En effet, comme pour l'emploi de "certes", c'est cette problématique qui soustend tous les autres passages au présent dans le roman. Tant répétée, la coïncidence devient inéluctable. Ainsi sont mis en relief dans "Été", la réticence d'Alphonsine face au départ de son ainé (p. 81-110), ce départ lui-même (p. 87-90), le présage du malheur entrevu par Alphonsine qu'elle associe à la mort d'Euchariste mais qui annonce en fait sa propre mort (p. 112) et la rupture affective d'Oguinase avec sa famille (p. 125). Dans "Automne" et "Hiver", ce sont la dernière visite d'Euchariste à son fils agonisant (p. 184), la décision du héros d'abandonner sa terre à Étienne (p. 230-231) et son arrivée chez Ephrem, triste début de l'exil (p. 238), que le présent vient réactualiser.

Les variations dans les rapports d'ordre reflètent la même logique. Genette désigne ainsi les écarts possibles dans un texte narratif entre l'ordre "réel" des événements racontés et l'ordre de leur présentation par le narrateur. Dans Trente arpents, la pré sentation choisie par l'auteur implicite est celle de l'ordre chronologique à quelques exceptions près. La décision d'Euchariste d'envoyer Oguinase au séminaire (p. 93-94), le départ d'Ephrem, fils préféré d'Euchariste après la mort de son ainé (p. 176-177) et l'acceptation résignée, par le héros, de l'inévitabilité de son propre exil (p. 229-230) sont présentés sous forme de retours en arrière. Chose intéressante, le rapprochement entre l'auteur implicite et son héros dicte non seulement les contextes qui déclenchent les écarts à l'égard du codage temporel ou des rapports d'ordre mais la nature même de l'écart choisi. Si les départs sont très souvent repoussés au passé, la mort réelle ou symbolique, rupture plus définitive, est toujours réactualisée, au moyen du récit ou présent.

Que conclure de notre analyse des modes de présence de l'auteur implicite dans Trente arpents? La première conclusion touche évidernment le rôle joué par l'auteur implicite vis-a-vis du lecteur visuel. Dans la première section du roman, les modes de présence retenus par l'auteur implicite confirment effectivement l'intention de documentaliste que lui a attribuée en général la critique. Mais ce n'est point le cas dans les sections subséquentes. Sur le plan des formes de présence, tant explicites que structurales, nous remarquọns alors un effacement progressif de l'auteur implicite au profit de la perspective de son personnage principal, Euchariste Moisan. Quittant son poste de sociologue qui souligne tout ce qui, dans la vie de Moisan, est typique du paysan de l'époque, l'auteur implicite finit par partager les conséquences affectives de la tragédie de son héros. Certes, rien n'empéche le lecteur "réel", par opposition à son homologue "virtuel", de faire une interprétation sociologique du sort de Moisan. II n'en reste pas moins vrai qu'au niveau non de l'interprétation mais du sens, pour reprendre la distinction de Torodov, ${ }^{19}$ nous avons affaire à un changement radical de 
perspective narrative. Qui plus est, ce changement de perspective nous permet d'isoler une problématique particulière, celle de la rupture, susceptible d'informer la construction du sens dans le roman à d'autres niveaux - thématique, événementiel, métaphorique, etc. ${ }^{20}$

Cette observation nous amène à deux autres conclusions qui sont moins des constatations que des points d'interrogation. La première concerne précisément la problématique de la rupture dans Trente arpents. L'importance de cette problématique est telle, et ce dans les moindres détails structuraux, que nous sommes tentée de nous interroger sur sa présence dans d'autres textes de Ringuet. S'agit-il d'un phénomène passager ou bien d'une constante dans l'oeuvre de Ringuet? Seule une analyse structuraliste des autres romans ringuetiens permettra de trancher la question.

Notre dernière observation porte sur la signification narrative, au sens large, du changement de perspective de l'auteur implicite. Se fondant sur une appréciation essentiellement intuitive, quelques critiques ont soutenu que, du moins sur le plan esthétique, la fin du roman paraît plus réussie que le début. ${ }^{21} \mathrm{Se}$ peut-il que cette impression soit due à l'effacement progressif de l'auteur implicite au profit de son personnage principal, c'est-z̀-dire, pour reprendre la terminologie de Lubbock, à l'abandon par l'auteur implicite de la narration (telling) en faveur de la dramatisation (showing)? La réponse affirmative qui s'impose nous oblige à reprendre, mais sur des bases plus rigoureuses, un aspect du moins des théories traditionnelles du point de vue. Or, si Lubbock et ses successeurs avaient le malheur de figer à la fois l'intention de l'auteur et le choix optimum des moyens requis pour atteindre les effets recherchés, frôlant parfois la recette de cuisine, ils n'avaient peut-être pas tort de vouloir ancrer un jugement esthétique sur des phénomènes narratifs précis. C'est justement l'opportunité et la pertinence d'une réarticulation, dans un contexte critique structuraliste, d'un tel ancrage, que vient affirmer notre analyse de Trente arpents.

1. Renaud, A. et Robidoux, R., le Roman canadien-français du vingtième siècle, Ottawa, Presses de l'Université d'Ottawa, 1966, p. 44; Viens, J., "la Terre" de Zola et "Trente arpents" de Ringuet, Montréal, Éditions Cosmos, 1970, p. 86-87.

2. Notons, entre autres, A. Pelletier et B. Brunet, cités par J. Viens, op. cit., p. 88.

3. Renaud A., et Robidoux, R., op. cit.

4. Servais-Maquoi, M., le Roman de la terre au Quebbec, Québec, Presses de l'Université Laval, 1974 , p. 154.

5. Voir, entre autres, Labonté, R., "Le paysage ringuetien", Voix et /mages du Pays, $1 \times(1975)$, p. 139-162 et "L'espace intérieur chez Ringuet", Canadian Literature, 71 (hiver 1976), p. 68-72; Socken, P., "The Narrative Structure of Trente arpents", Canadian Literature, 86 lautomne 1980), p. 152-156; Hoekema, H., "lllusion of realism in Thirty Acres", Essays in Canadian Writing, 17 (printemps 1980), p. 102-112.

6. La distinction, qui n'est pas respectée par les critiques qui insistent sur l'objectivité de Ringuet, est de Wayne Booth, The Rhetoric of Fiction, Chicago et Londres, University of Chicago Press, 1961, p. 71.77.

7. Hid., p. 16-20.

8. A l'instar de T. Todorov ("Les catégories du récit littéraire", Communications, 8 (1966), p. 126), nous allons considérer que ces deux instances sont identiques.

9. Van Rossum-Guyon, F., "Point de vue ou perspective narrative", Poetique, 4 (1970), p. 479. 
10. Genette, G., Figures III, Paris, Editions du Seuil, 1972, p. 77-144.

11. Ringuet, Trente Arpents, Paris, Flammarion, 1938, p. 51. Les références étant toutes tirés de cette édition, nous indiquerons, désormais, la pagination entre parenthèses.

12. R. Labonté signale aussi cet aspect universalisant des descriptions de Ringuet, sans pourtant l'analyser systématiquement tout au long du texte ("Le paysage ringuétien", Voix et Images du Pays, IX (1975), p. 152).

13. Tynianov, J., "De l'évolution litteraire" in Torodov, T., (rééd.), Theorie de la litterature, Paris, Editions du Seuil, 1965, p. 125.

14. Genette, G., op. cit., p. 123.

15. Viens arrive à des chiffres differents: Eté, 17 ans; Automne, 11 ans; Hiver, 3 ans et demi lop. cit., p. 1021. Le rapport entre les durées des différentes sections reste néanmoins à peu près le mème.

16. Genette, G., op. cit., p. 229.

17. Le présent historique s'emploie en général dans un contexte de temps passés (imparfait, plus-que-parfait) ce qui n'est pas le cas dans les passages racontés au présent dans Trente Arpents. (Weinrich, H., Je Temos, Paris, Seuil, 1973, p. 296.

18. Ibid., p. 39-49.

19. Todorov, T., loc, cit., p. 125.

20. L'importance thématique du départ et de l'abime a été soulignée par quelques critiques: Labonté, R., "L'espace intérieur chez Ringuet", loc. cit.; Renaud, A., et Robidoux, R., op. cit.

21. Viens, J., op. cit., p. 103. Remarquons que Viens, en plus de faire allusion au jugement d'autres critiques, fonde le sien sur le décalage entre la durée narrative des premiéres sections du roman et celle de la dernière, "Hiver". 